\title{
Depletion-interaction effects on the tunneling conductivity of nanorod suspensions
}

\author{
B. Nigro, ${ }^{1}$ C. Grimaldi, ${ }^{2}$ M. A. Miller, ${ }^{3}$ P. Ryser, ${ }^{1}$ and T. Schilling ${ }^{4}$ \\ ${ }^{1}$ LPM, Ecole Polytechnique Fédérale de Lausanne, Station 17, Case Postale 1015 Lausanne, Switzerland \\ ${ }^{2}$ Laboratory of Physics of Complex Matter, Ecole Polytechnique Fédérale de Lausanne, Station 3, Case Postale 1015 Lausanne, Switzerland \\ ${ }^{3}$ Department of Chemistry, Durham University, South Road, Durham DH1 3LE, United Kingdom \\ ${ }^{4}$ Université du Luxembourg, 162 A, Avenue de la Faïencerie, L-1511 Luxembourg
}

(Received 8 August 2013; revised manuscript received 25 September 2013; published 25 October 2013)

\begin{abstract}
We study by simulation and theory how the addition of insulating spherical particles affects the conductivity of fluids of conducting rods, modeled by spherocylinders. The electrical connections are implemented as tunneling processes, leading to a more detailed and realistic description than a discontinuous percolation approach. We find that the spheres enhance the tunneling conductivity for a given concentration of rods and that the enhancement increases with rod concentration into the regime where the conducting network is well established. By reformulating the network of rods using a critical path analysis, we quantify the effect of depletion-induced attraction between the rods due to the spheres. Furthermore, we show that our conductivity data are quantitatively reproduced by an effective-medium approximation, which explicitly relates the system tunneling conductance to the structure of the rod-sphere fluid.
\end{abstract}

DOI: 10.1103/PhysRevE.88.042140

PACS number(s): 64.60.ah, 73.40.Gk, 61.46.Fg, 72.80.Tm

\section{INTRODUCTION}

Electrical conductivity in composite materials can be achieved by embedding a network of conducting filler particles in an insulating polymeric matrix. It is well known that the conductivity of such dispersions can be greatly enhanced by using filler particles with a large degree of shape anisotropy, due to the increased excluded-volume effects associated with highly nonspherical particles [1,2]. This enhancement in turn makes it possible to reduce significantly the loading of conductor necessary to establish an adequate level of electrical connectedness for a given purpose [3-8].

A low filler concentration is often desirable in order to preserve the optical and mechanical properties of the host insulating medium. Therefore, the rationale behind a large amount of experimental work done in the last decade, with particular emphasis on carbon nanotube (CNT) fillers dispersed in polymeric matrices, has been to attain high conductivities with low filler concentration (see, for example, Refs. [9,10] for comprehensive reviews). With this goal in mind, elongated fillers with diameters on the micron scale, such as stainless steel fibers or metallized glass fibers, were already being employed in the early 1980s [8]. In nanocomposites, in addition to CNTs, insulating polymers have also been loaded with short conducting carbon fibers [11] and metal nanowires $[12,13]$.

Recently it has been reported that the conductivity of a CNT-based nanocomposite can be greatly enhanced at a given concentration of CNTs by adding small quantities of conductive latex depletants, which lead to attractive depletion interactions between the CNTs [14]. Weak attractive interactions induced by surfactant micelles have also been shown to strongly enhance the dielectric constant in dispersions of CNTs in aqueous solution [15].

On the theoretical side, by modeling fibrous fillers as rigid cylinders, capped cylinders, or spheroids, conductivity has usually been interpreted in terms of percolation theory using both analytical $[1,2,5,16,17]$ and numerical [18-21] methods. In the percolation approach, the filler particles are considered as being coated with a penetrable contact shell, and connectivity between two particles is established at the point where their shells overlap. The system becomes electrically conducting at the percolation threshold $\phi_{c}$, which is the critical volume fraction of filler particles above which a system-spanning cluster of interconnected fillers is always found. The calculated values of $\phi_{c}$ are thus used to estimate the conductor-insulator transition of real composites.

The theoretical studies mentioned above deal with dispersions of penetrable or impenetrable pure rodlike fluids without taking into account further sources of interaction. In the framework of percolation theory, the effects of depletion interactions have been considered in Ref. [3], where particle interactions are mediated by a square-well attractive potential and in Refs. [7,14,22], where hard spherical depletant particles are added explicitly into the insulating phase. This work has revealed an interplay of opposing effects. On the one hand, depletion encourages attraction between the surfaces of the rods, which leads to a higher density of overlapping shells and a lower percolation density. On the other hand, depletion also enhances mutual alignment of neighboring rods, decreasing the spatial extent of the clusters and tending to raise the percolation threshold. However, the former effect has been shown to be the stronger [22] and the net result of depletion is to lower the density required for percolation.

Theoretical studies on the conductivity of networks of attractive rods have so far concentrated on determining the location of the percolation threshold for a particular choice of contact shell thickness. For a real composite, however, the details of charge transport across the network at filler concentrations above the conductor-insulator transition are crucial. Motivated by the recent reinterpretation of tunneling transport for composites loaded with anisotropic particles in terms of a global tunneling network [23], we present here a numerical analysis of the conductivity of mixtures composed of conducting spherocylinders and insulating spheres. We show that the addition of a low concentration of spherical depletants enhances the tunneling conductivity of the subsystem 
of conducting rods with respect to the case without depletants. The magnitude of this effect rises as the concentration of rods is increased and continues to enhance the conductivity in the regime where the conducting network is well established, eventually falling off slightly at the highest concentrations included in this work. By computing the critical distance $\delta_{c}$ associated with the critical path approximation for system conductivity, we observe that the average of the relevant interparticle distance is lowered by depletion, thereby favoring electronic transport.

We also show that the numerical conductivity results can be reproduced by applying an effective-medium approximation, previously formulated for the case of spherical fillers, here generalized to the case of fluids of conducting spherocylinders. We find that the behavior of the effective conductance and the effects of the depletant particles can be fully understood in terms of an appropriate pair distribution function dependent on the distance between the cores of two spherocylinders.

\section{MODEL AND SIMULATIONS}

We model mixtures of conducting rodlike fillers and insulating spherical depletants by a binary system composed of $N_{r}$ impenetrable spherocylinders of length $L$ and diameter $D$, and $N_{s}$ hard spheres. Since in experiments the depletant particles have diameters comparable to those of CNTs [15], we set the sphere diameter equal to $D$. We also require that the two species of particles are mutually impenetrable. By representing the spherical depletants explicitly, rather than implicitly through an effective potential, we ensure that all many-body effects are, by construction, included. The volume fractions for spherocylinders and spheres dispersed in a cubic box of edge $\mathcal{L}$ are, respectively, $\phi_{r}=\rho_{r} v_{r}$ and $\phi_{s}=\rho_{s} v_{s}$. Here, $v_{r}=(\pi / 6) D^{3}+(\pi / 4) L D^{2}$ and $v_{s}=(\pi / 6) D^{3}$ are the volumes of one spherocylinder and one sphere, respectively, while $\rho_{r}=N_{r} / \mathcal{L}^{3}$ and $\rho_{s}=N_{s} / \mathcal{L}^{3}$ are the corresponding number densities. We simulate systems of $1000 \leqslant N_{r} \leqslant 2888$ sphereocylinders with $L / D=5(\mathcal{L} \geqslant 3 L)$ and $1000 \leqslant N_{r} \leqslant$ 2780 spherocylinders with $L / D=10(\mathcal{L} \geqslant 4 L)$. Depending on $\phi_{s}$, the number of spheres ranges between $N_{s}=8500$ and $N_{s}=3.2 \times 10^{5}$.

To generate equilibrium dispersions of the mixture, we start by introducing the rods and the spheres into the periodic simulation cell by random sequential addition, i.e., simply assigning a uniformly distributed random position and orientation to each particle in turn and accepting any insertion that does not lead to an overlap with particles that have been already placed. We test the overlap between two spherocylinders $i$ and $j$ by computing the shortest distance $d_{i j}$ between the two line segments coinciding with the axes of the spherocylinders [24]. If $d_{i j}$ is less than $D$, the two spherocylinders overlap. The shortest distance is computed by minimizing $\left|\mathbf{r}_{i j}+\lambda_{i} \hat{\mathbf{u}}_{i}-\lambda_{j} \hat{\mathbf{u}}_{j}\right|^{2}$ with respect to $\lambda_{i}$ and $\lambda_{j}$ in the range $-L / 2$ to $L / 2$, where $\mathbf{r}_{i j}$ is the displacement vector between the two rod centers, and $\hat{\mathbf{u}}_{i}$ and $\hat{\mathbf{u}}_{j}$ are unit vectors along the axes of rods $i$ and $j$, respectively (see Fig. 1). We then perform a standard Metropolis Monte Carlo equilibration consisting of trial translational and rotational moves for each rod and sphere. Given the simple hard-core potential, trial

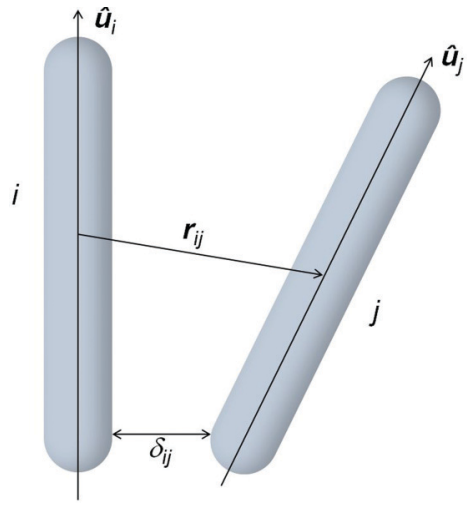

FIG. 1. (Color online) Schematic representation of two spherocylinders. The position vector $\mathbf{r}_{i j}$ identifies the position of the center of mass of rod $j$ with respect to the center of mass of $\operatorname{rod} i$. $\hat{\mathbf{u}}_{i}$ and $\hat{\mathbf{u}}_{j}$ are unit vectors along the axes of rods $i$ and $j$, respectively. $\delta_{i j}$ is the shortest distance between the surfaces of the two rods.

moves that do not lead to hard-core overlap of the particles are always accepted, while moves that do generate overlaps are rejected. We monitor any global alignment of the rods by means of the nematic order parameter $S$ which is the largest eigenvalue of the tensor $\mathbf{Q}=\left(2 N_{r}\right)^{-1} \sum_{i}^{N_{r}}\left(3 \hat{\mathbf{u}}_{i} \hat{\mathbf{u}}_{i}-\mathbf{1}\right)$, where $\mathbf{1}$ is the identity matrix [25]. For the range of concentrations of rods and spheres used in this study, $S$ is always close to zero, which indicates that the dispersions of spherocylinders are isotropic.

In modeling the electron transfer between spherocylinders, we neglect charging and Coulomb interaction effects and assume that the conductance $g_{i j}$ between any two spherocylinders $i$ and $j$ is given by single-electron tunneling processes:

$$
g\left(\delta_{i j}\right)=g_{0} \exp \left(-\frac{2 \delta_{i j}}{\xi}\right) .
$$

Here, $\delta_{i j}$ is a shorthand notation for $\delta_{i j}=\delta_{i j}\left(\mathbf{r}_{i j} ; \hat{\mathbf{u}}_{i}, \hat{\mathbf{u}}_{j}\right)$ which is the minimal distance between the surfaces of rods $i$ and $j$, given their relative position and orientation vectors, as schematically illustrated in Fig. 1. The tunneling decay length $\xi$ depends on the electronic potential barrier between two spherocylinders and its value ranges from a fraction of a nanometer to a few nanometers. In Eq. (1) we set the conductance prefactor $g_{0}$ equal to unity, so that the conductance between two touching spherocylinders is $g(0)=1$.

Equation (1) captures the dominant dependence of the conductance between two rods on their relative positions and orientations, i.e., the exponential decay as a function of the single distance of closest approach $\delta_{i j}$ of the rods. However, this simple formula neglects any explicit dependence of the conductivity on the mutual alignment of the rods. The relative orientation does not affect the exponential dependence on $\delta_{i j}$, but strong alignment can enhance the preexponential factor. This enhancement becomes significant only when the centers of the two rods are close and the angle between the two rods is small. A detailed analysis of the pairwise conductivity of rods will be the subject of a separate presentation [26]. 


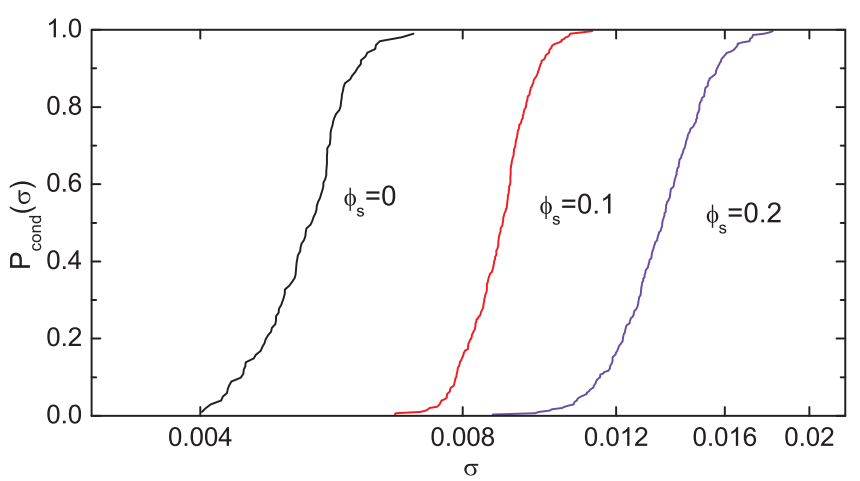

FIG. 2. (Color online) The cumulative conductivity distribution $P_{\text {cond }}(\sigma)$ for systems of conducting spherocylinders of aspect ratio $L / D=5$ and volume fraction $\phi_{r}=0.135$ mixed with different concentrations $\phi_{s}$ of insulating spherical particles. The tunneling decay length is fixed at $\xi / D=0.2$.

\section{NETWORK CONDUCTIVITY}

To obtain the overall conductivity $\sigma$, we construct for each realization of the system a conductance network by assigning the tunneling conductances from Eq. (1) to each pair of spherocylinders. The tunneling network does not include the spheres, which are insulating. The effect of the spheres on conductivity is indirect, through the depletion forces that they induce between the spherocylinders.

Despite involving only the spherocylinders, the network is composed of $N_{r}\left(N_{r}-1\right) / 2$ tunneling bonds, which renders the numerical solution of the overall conductivity computationally demanding for the system sizes considered here. We therefore reduce the number of bonds, and so the coordination number of each node, by eliminating from the network the tunneling conductances associated with pairs of spherocylinders that are sufficiently far apart not to contribute to the overall conductivity $[27,28]$.

We solve the Kirchhoff equations of the reduced network by combining exact numerical decimation with a preconditioned conjugate gradient algorithm [29-32]. The decimation algorithm uses exact transformations to eliminate nodes from the network and to update the conductances adjacent to the eliminated nodes [29], as discussed in the Appendix. We iteratively decimate the network starting from the nodes with the lowest coordination number until a single conductance is left, whose value coincides with the conductance of the original network. For configurations such that the computational time for the node decimation is too large, we switch to the conjugate gradient method with Cholesky preconditioning (see, e.g., Refs. [30,31]) applied to the partially decimated network. We have applied this procedure to 300 realizations of each system. For each equilibrium configuration we obtain the conductivity from $\sigma=G D / \mathcal{L}$, where $G$ is the conductance of the reduced network for a unit voltage drop applied to two opposite faces of the simulation box. From the sample of configurations at a given combination of $\phi_{r}, \phi_{s}, \xi / D$, and $L / D$, we construct the cumulative conductivity distribution function $P_{\text {cond }}(\sigma)$, which gives the probability of finding conductivities less than or equal to $\sigma$ over all realizations. This function is shown in Fig. 2 for three concentrations of depletants with rods of aspect ratio
$L / D=5$, packing fraction $\phi_{r}=0.135$, and tunneling length $\xi / D=0.2$.

To compare conductivities at different concentrations of spheres or rods, we concentrate on the conductivity $\sigma_{m}$ that marks the midpoint of the cumulative distribution, $P_{\text {cond }}\left(\sigma_{m}\right)=$ $1 / 2 . \sigma_{m}$ increases with the concentration of depletant spheres, as seen by comparing the results for $\phi_{s}=0,0.1$, and 0.2 shown in Fig. 2. This trend is confirmed in Fig. 3(a), where we show $\sigma_{m}$ as a function of the rod volume fraction $\phi_{r}$ for $L / D=5$ and $L / D=10$ and for different depletant concentrations $\phi_{s}$. For a given $\phi_{s}$, the enhancement of $\sigma_{m}$ is negligible at low rod concentrations $\phi_{r}$, where small changes in the fluid structure have little effect on network connectivity, and increases with rod concentration as the network emerges. In this regime, the increase is such that systems with $L / D=5$ and $\phi_{s}=0.2$ have conductivities approaching those of hard rods with $L / D=$ 10 and no depletants. There is a slight drop in the level of enhancement at the very largest rod concentrations, since even a network of pure rods is highly ramified at such density. For given densities of both rods and spheres, the depletion-induced increase of $\sigma_{m}$ becomes stronger as the tunneling decay length decreases, as seen by comparing the results for $\xi / D=0.2$ and 0.1 shown in Fig. 3(b).

We note that in our calculations we neglect any contribution stemming from intrinsic conductivity $\sigma_{\text {ins }}$ of the insulating matrix, which in real composites is small but finite at nonzero temperatures. The inclusion of $\sigma_{\text {ins }}$ would prevent the conductivity of the composite system from dropping to zero as $\phi_{r} \rightarrow 0$ by limiting its value at $\sigma_{\text {ins }}$. In this respect, the conductor-insulator transition point can be estimated by the value of $\phi_{r}$ such that $\sigma_{m} \approx \sigma_{\text {ins }}[23,28]$.

To explore the mechanism causing the conductivity enhancement highlighted in Fig. 3, we calculate the critical distance $\delta_{c}$, which is defined as the smallest distance such that the subnetwork of bonds satisfying $\delta_{i j} \leqslant \delta_{c}$ still spans the entire sample. The interest in evaluating $\delta_{c}$ stems from the observation of Ref. [33] that when the values of the single conductances $g\left(\delta_{i j}\right)$ vary over many orders of magnitude, and so when the $\delta_{i j}$ distances span several multiples of the tunneling decay length $\xi$, the network conductance is dominated by the characteristic bond conductance $g\left(\delta_{c}\right)=$ $g_{0} \exp \left(-2 \delta_{c} / \xi\right)$. In this situation, the network conductivity is well estimated by the critical path approximation (CPA) formula [33]:

$$
\sigma_{\mathrm{cpa}}=\sigma_{0} \exp \left(-\frac{2 \delta_{c}}{\xi}\right)
$$

where $\sigma_{0}$ is a slowly varying function of $\delta_{c}$ and can be considered constant. Using this expression, we can represent the conductivity as a function of $\delta_{c}$, thereby making a connection with approaches based on percolation theory. However, unlike the usual percolation approach of imposing a fixed contact shell thickness, the CPA uses a variable thickness whose value responds to the structure of the fluid. It follows that Eq. (2) predicts through $\delta_{c}$ an implicit dependence of the conductivity on the parameters of the network of conducting particles, in contrast to the usual percolation approach in which the information on the fluid structure is contained only in the percolation threshold $\phi_{c}$. 

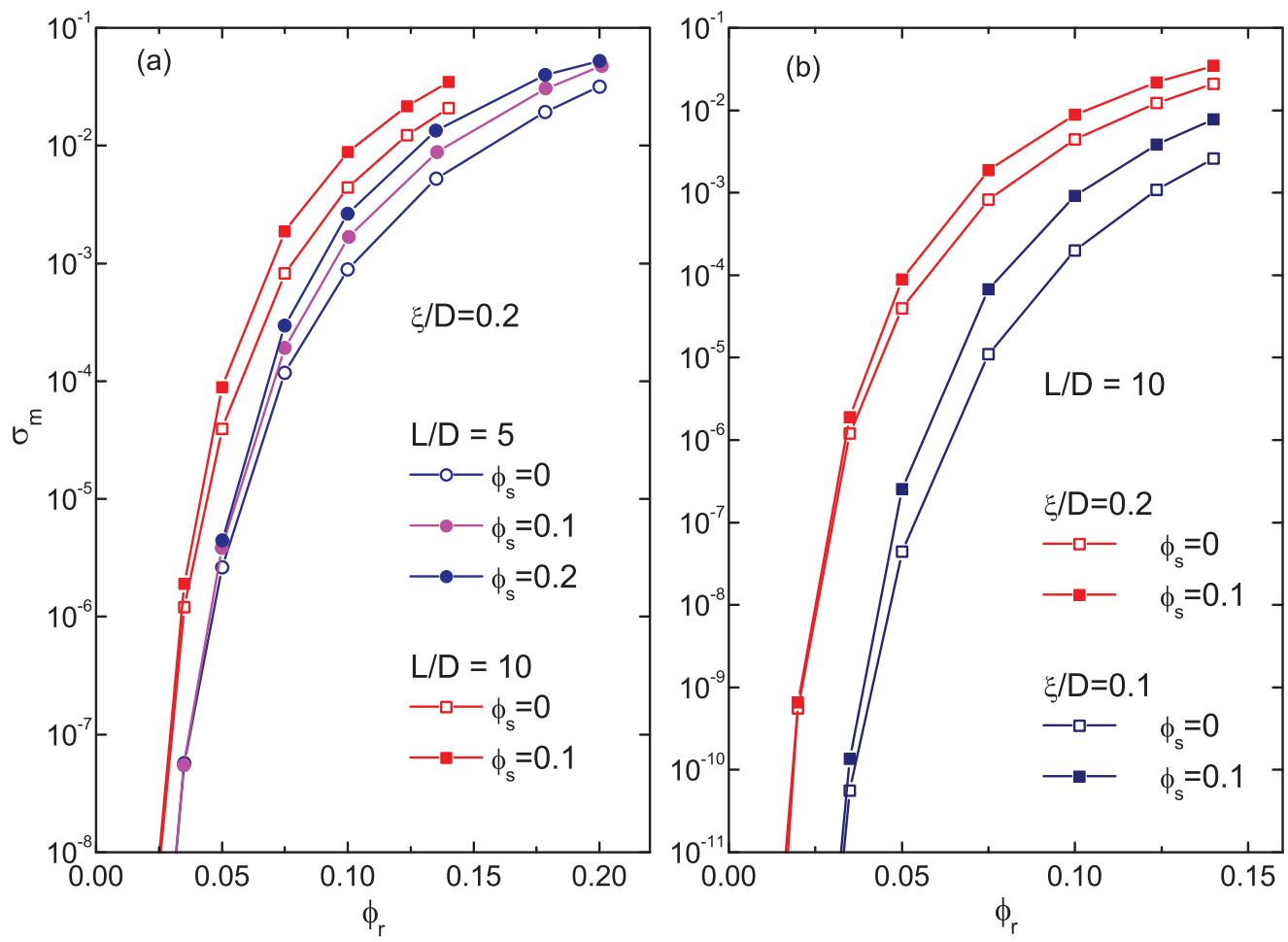

FIG. 3. (Color online) Conductivity $\sigma_{m}$ computed from the numerical solution of the tunneling network equations as a function of the volume fraction $\phi_{r}$ of conducting spherocylinders and for different concentrations $\phi_{s}$ of insulating spheres. Results for (a) $\xi / D=0.2$ and $L / D=5,10$ and (b) $L / D=10$ and $\xi / D=0.1,0.2$.

We calculate $\delta_{c}$ following the method described in Refs. [27,28,34,35], which consists of constructing the percolation probability $P_{\text {perc }}(\delta)$ from the values of the percolation distance $\delta$ calculated for all realizations of the system with a given combination of rod and sphere concentrations. From the condition $P_{\text {perc }}\left(\delta_{c}\right)=1 / 2$, which provides a robust estimate of the critical distance [28], we find that the effect of the depletant particles is to lower $\delta_{c}$ compared to the case without depletants $\left(\phi_{s}=0\right)$, as shown in Figs. 4(a) and 4(b). Furthermore, this lowering becomes asymptotically negligible as the concentration of spherocylinders goes to zero.

The behavior of $\delta_{c}$ shown in Fig. 4 can be understood in terms of the hard-core repulsion between rods and spheres. When two spherocylinders approach closely, the volume available to spheres increases, inducing an effective attraction between the spherocylinders. In this way, percolation of the system of rods is established for shorter distances compared to the case without spheres, thus reducing $\delta_{c}$.

By combining the results in Fig. 4 with the CPA formula given in Eq. (2), we see that the lowering of $\delta_{c}$ induced by the depletant spheres directly translates into a corresponding enhancement of $\sigma_{\mathrm{cpa}}$. By identifying $\sigma_{\mathrm{cpa}}$ with $\sigma_{m}$, the depletion interaction effect thus explains the enhancement of the sample conductivity shown in Fig. 3. To test the accuracy of the CPA conductivity, we plot in Fig. 5 the calculated $\ln \left(\sigma_{m}\right)$ values of Fig. 3 as a function of $\delta_{c} / D$ (symbols) and compare them with Eq. (2) using $\sigma_{0}=0.026$ (dashed lines), obtained from a fit at low conductivity. The exponential behavior of $\sigma_{\mathrm{cpa}}$ reproduces quantitatively the functional dependence of $\sigma_{m}$ for $\delta_{c} / D \gtrsim$
0.5 , while the CPA becomes less accurate as $\delta_{c} / D \rightarrow 0$. In general, the CPA is not expected to reproduce correctly the tunneling conductivity when $\delta_{c} \lesssim \xi$ since in this case the $\delta_{i j}$ distances would not be widely distributed with respect to $\xi$, as previously discussed. Although $\sigma_{m}$ deviates from a simple exponential function of $\delta_{c}$ at low $\delta_{c}$, data for different aspect ratios and depletant concentrations still collapse onto the same curve for a given tunneling length $\xi$, as shown by the two sets of data for different $\xi / D$ in Fig. 5.
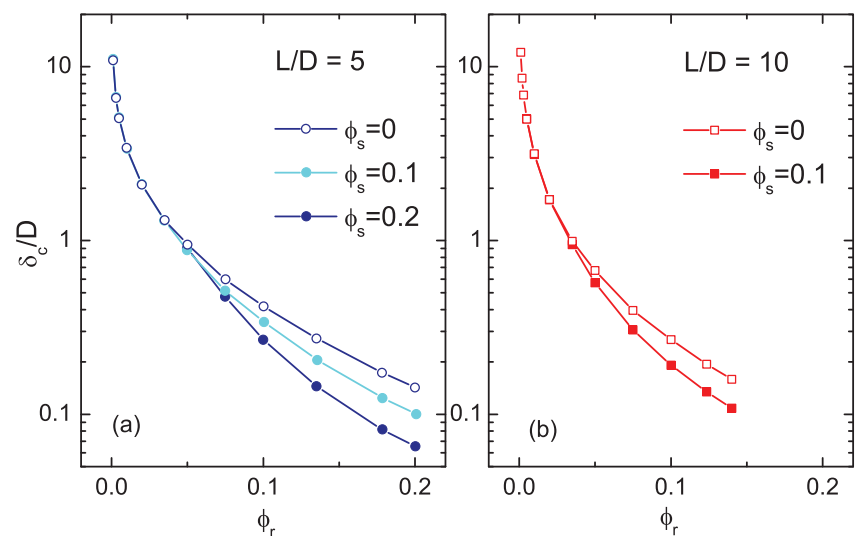

FIG. 4. (Color online) Critical distance $\delta_{c}$ as a function of the volume fraction $\phi_{r}$ of spherocylinders with (a) $L / D=5$ and (b) $L / D=10$, and for different volume fractions $\phi_{s}$ of the depletant spheres. 


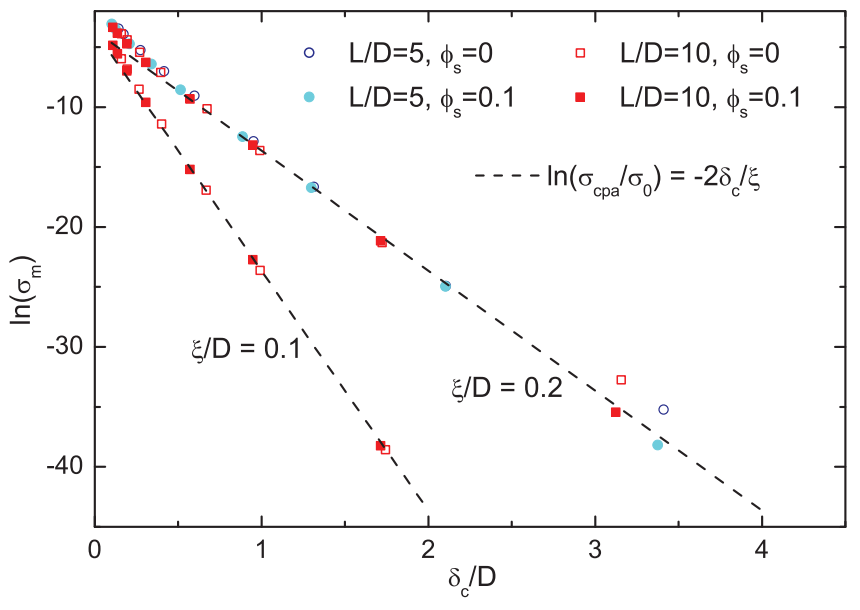

FIG. 5. (Color online) Conductivity values of Fig. 3 replotted as a function of $\delta_{c} / D$, where $\delta_{c}$ is the calculated critical distance. The dashed lines are linear fits to Eq. (2). For all cases the prefactor $\sigma_{0}$ is 0.026 .

\section{EFFECTIVE-MEDIUM APPROXIMATION}

An alternative approach to the CPA to describe tunneling transport in suspensions of conducting particles is provided by the effective-medium approximation (EMA) applied to the network of tunneling conductances of Eq. (1). In contrast to the CPA, the EMA approach does not rely on the definition of a percolation quantity such as $\delta_{c}$, and applies also to systems with typical interparticle distances lower than the tunneling decay length $\xi$. Furthermore, within the two-site approximation, the EMA explicitly relates the system conductance to the pair distribution function of the conducting particles, thus emphasizing the role of the fluid structure in tunneling transport.

We apply the EMA to the fluid of conducting rods and spherical depletants following the method described in Ref. [36], in which each pairwise conductance $g\left(\delta_{i j}\right)$ in the network of $N_{r}$ spherocylinders is replaced by an effective conductance $\bar{g}$ that is independent of $\delta_{i j}$. Requiring equivalence between the average resistance of the original tunneling network and that of the effective one, the conductance $g^{*}=N_{r} \bar{g} / 2$ between any two nodes of the network is calculated from the solution of the following equation:

$$
\frac{1}{N_{r}}\left\langle\sum_{i=1}^{N_{r}} \sum_{j \neq i}^{N_{r}} \frac{g\left(\delta_{i j}\right)}{g^{*}+g\left(\delta_{i j}\right)}\right\rangle=2,
$$

where the angular brackets indicate an ensemble average over configurations. The two-point EMA conductance $g^{*}$ is independent of the network size, and can thus be regarded as a measure of the system conductivity. This size independence is a consequence of the completeness of the EMA network, in which all nodes are connected with identical resistances [37].

Since the tunneling conductances depend only on the distances $\delta_{i j}$ between the spherocylinders, we recast Eq. (3) in a form involving the probability function of the distance between pairs of rods, $P_{\text {pair }}(\delta)$, which is readily obtained by

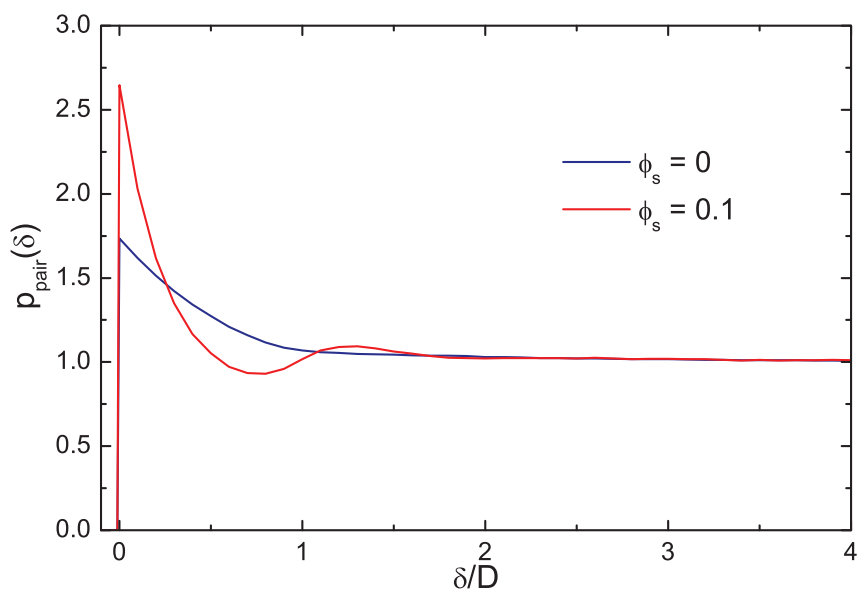

FIG. 6. (Color online) The distribution function of distance $\delta$ between the surfaces of pairs of spherocylinders relative to the ideal case, $p_{\text {pair }}(\delta)$, for aspect ratio $L / D=10$ at $\phi_{r}=0.14$ as a function of $\delta / D$.

binning of $\delta_{i j}$ during the simulations:

$$
\rho_{r} \int_{0}^{\infty} d \delta \frac{P_{\text {pair }}(\delta)}{\left(g^{*} / g_{0}\right) \exp (2 \delta / \xi)+1}=2 .
$$

The function $P_{\text {pair }}(\delta)$ in our mixture of hard-core particles is best visualized by normalizing it with respect to the corresponding function $P_{\mathrm{id}}(\delta)$ of ideal (fully penetrable) spherocylinders at the same density. The resulting quantity $p_{\text {pair }}(\delta)=P_{\text {pair }}(\delta) / P_{\text {id }}(\delta)$ coincides with the average correlation function introduced in Ref. [38]. The ideal distribution can be derived from

$$
P_{\mathrm{id}}(\delta)=\rho_{r} S_{\mathrm{exc}}(\delta),
$$

where $S_{\text {exc }}(\delta)$ is the surface area of the orientationally averaged excluded volume $V_{\mathrm{exc}}(\delta)$ for two rods with cores constrained to a fixed closest distance $\delta[38,39]$. Hence, $S_{\mathrm{exc}}(\delta)=d V_{\mathrm{exc}}(\delta) / d \delta$, where [1]

$$
V_{\mathrm{exc}}(\delta)=\frac{4 \pi}{3}(D+\delta)^{3}+2 \pi(D+\delta)^{2} L+\frac{\pi}{2}(D+\delta) L^{2} .
$$

In Fig. 6 we show the numerical $p_{\text {pair }}(\delta)=P_{\text {pair }}(\delta) / P_{\text {id }}(\delta)$ averaged over 300 realizations for $\phi_{r}=0.14, L / D=10$ with $\phi_{s}=0$ and 0.1 . The effect of the depletants is manifest in the increased oscillations of $p_{\text {pair }}(\delta)$ [22] for the case $\phi_{s}=0.1$, indicating a strong spatial correlation due to the reduction of the interparticle distances.

Using the measured $P_{\text {pair }}(\delta)$ to solve Eq. (4) numerically for $g^{*}$, we obtain the plots in Fig. 7. The increased population of rods at short distances, induced by the depletant spheres and highlighted in Fig. 6, promotes tunneling processes at short distances, which result in larger EMA conductances $g^{*}$ compared to the depletant-free case. Figure 7 indeed shows $g^{*}$ values systematically enhanced by the introduction of depletants for fixed $\phi_{r}$ and $L / D$. Furthermore, the EMA results in Fig. 7 reproduce accurately the conductivity behavior obtained by the full numerical solution of the tunneling network shown in Fig. 3. 

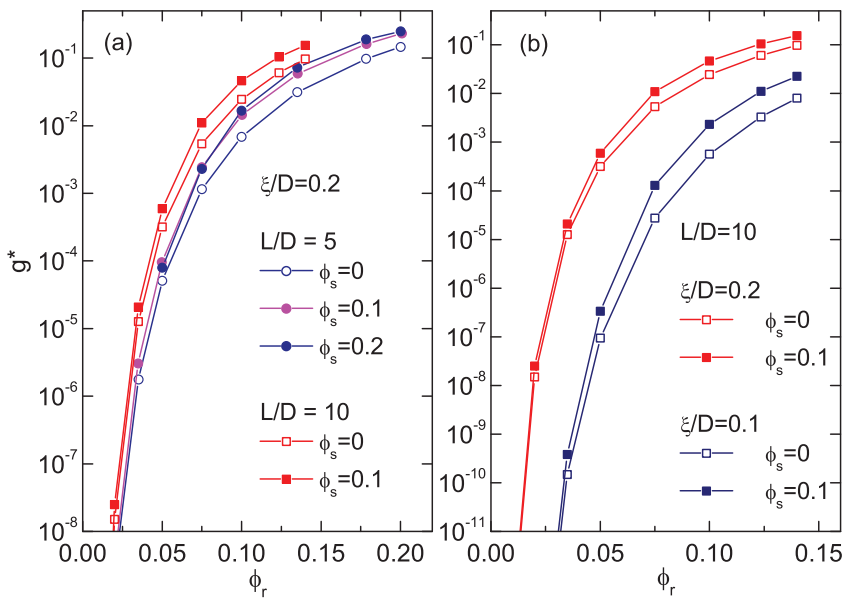

FIG. 7. (Color online) Two-point EMA conductance $g^{*}$ obtained from the numerical solution of Eq. (4) as a function of $\phi_{r}$ and for different volume fractions $\phi_{s}$ of insulating spheres. Results for (a) $\xi / D=0.2$ and $L / D=5,10$, and (b) $L / D=10$ and $\xi / D=0.1,0.2$.

\section{CONCLUSIONS}

Our simulation results show that the tunneling conductivity of fluids of rods is systematically enhanced by the addition of insulating spheres, even in the regime where the conducting network is well established. The mechanism of transport enhancement is the depletion effect of the spheres, which induces an effective attraction between the conducting rods.

Previous work $[3,7,14,22]$ has already established that the percolation threshold with an arbitrarily chosen contact shell thickness is lowered by depletion attraction. However, we have taken the analysis further in the present work in two ways. First, within the framework of percolation theory, the critical path approximation not only shows and quantifies how the minimum distance required for a subsystem of rods to span the system decreases with increasing depletant concentration, but also provides a good approximation to the conductivity over a wide range of parameters, thus clarifying the role of depletants through the reduction of the dominant tunneling distances.

Second, moving on from approaches based on percolation theory, we were also able to reproduce the simulation-based conductivity results by applying a simple effective-medium approximation to the tunneling network formed by the rods. Using the pair distribution function for the distance of closest approach of the spherocylinders, computed from our simulations, we found that the resulting effective conductance has the same functional dependence on the system parameters as was observed from the full calculation of the conductivity.

To conclude, let us comment on a possible extension of our work. As pointed out in Sec. II, the model of electron transfer of Eq. (1) neglects possible effects of mutual rod alignment on the probability of tunneling between rods. Although this approximation is valid for isotropic dispersions of rods, such as the ones considered in the present work, for systems with a high degree of rod alignment the dependence of tunneling on the relative rod orientation should be taken into consideration [26]. In particular, the tunneling conductance is expected to be larger when the rods are aligned, and so it may compete with the reduction of connectivity when the concentration of depletants is sufficiently large to induce nematic order. Further studies in this direction are computationally demanding, but would shed light on still unexplored issues.

\section{ACKNOWLEDGMENT}

B.N. acknowledges support by the Swiss National Science Foundation (Grant No. 200020-135491).

\section{APPENDIX: ALGORITHM FOR DECIMATION OF CONDUCTANCE NETWORKS}

The decimation algorithm [29] is based on the star-mesh transformation, well known in electrical circuit theory, which eliminates one node from the network at the price of inserting new conductances between certain remaining nodes. The new conductances are chosen so as to ensure electrical equivalence between the modified and the original networks. If the node $i$ is directly connected to $n$ other nodes through conductances $g_{i l}$, the transformation eliminates the node $i$ and inserts $n(n-1) / 2$ new conductances between each pair of the nodes which were originally connected to $i$. If one such pair has node indices $j$ and $k$, the new conductance is

$$
g_{j k}=\frac{g_{i j} g_{i k}}{\sum_{l} g_{i l}}
$$

where the sum runs over all neighbors $l$ of node $i$. If the original network had pairs of neighbors already connected, the new conductances are inserted in parallel to the existing ones. An illustration of the star-mesh transformation for $n=3$ is shown in Fig. 8.

We decimate the network by sequentially applying the star-mesh transformation to all nodes except those representing the electrodes. Ideally, by iterative decimation, the whole network is replaced by just one conductance between the electrodes, which is the equivalent conductance of the network. However, for networks with high degrees of coordination per node, as in systems with large concentrations of rods, the decimation procedure increases enormously the number of added conductances as the nodes are eliminated, considerably increasing the computational time. In this case, we implement the conjugate gradient method with Cholesky preconditioning [30,31], which performs better when applied to resistor networks with large coordination numbers.

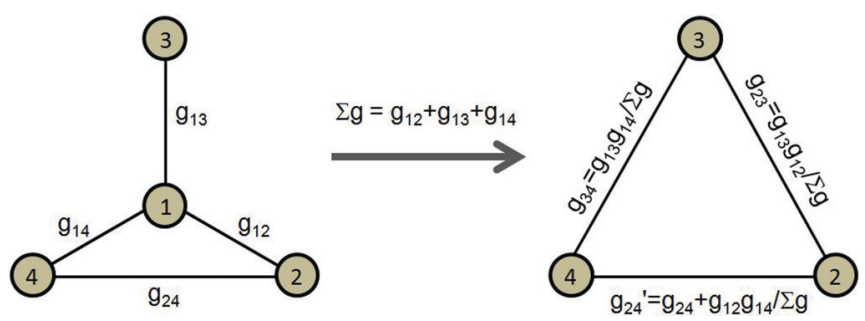

FIG. 8. (Color online) Schematic illustration adapted from Ref. [32] of the star-mesh transformation applied to a network with three terminal nodes. The transformation acts by (i) removing the internal node 1 and the conductances between the nodes 2,3 , and 4 , and (ii) inserting new conductances between the pairs $(2,3),(2,4)$, and $(3,4)$. The new conductance between 2 and 4 is added in parallel to the conductance $g_{24}$ of the original network. 
[1] I. Balberg, C. H. Anderson, S. Alexander, and N. Wagner, Phys. Rev. B 30, 3933 (1984).

[2] A. L. R. Bug, S. A. Safran, and I. Webman, Phys. Rev. Lett. 54, 1412 (1985).

[3] A. V. Kyrylyuk and P. van der Schoot, Proc. Natl. Acad. Sci. USA 105, 8221 (2008).

[4] T. Schilling, S. Jungblut, and M. A. Miller, in Handbook of Nanophysics: Nanotubes and Nanowires (Taylor \& Francis, New York, 2010).

[5] K. Leung and D. Chandler, J. Stat. Phys. 63, 837 (1991).

[6] A. P. Chatterjee, J. Chem. Phys. 113, 9310 (2000).

[7] X. Wang and A. P. Chatterjee, J. Chem. Phys. 118, 10787 (2003).

[8] R. Crossman, Polym. Eng. Sci. 25, 507 (1985).

[9] W. Bauhofer and J. Z. Kovacs, Compos. Sci. Technol. 69, 1486 (2009).

[10] M. S. Dresselhaus, G. Dresselhaus, and P. Avouris, Carbon Nanotubes: Synthesis, Structure, Properties and Applications (Springer, New York, 2011).

[11] M. H. Al Saleh and U. Sundararaj, Carbon 47, 2 (2009).

[12] G. A. Gelves, B. Lin, U. Sundararaj, and J. A. Haber, Adv. Funct. Mater. 16, 2423 (2006).

[13] M. Weber and M. R. Kamal, Polym. Compos. 18, 726 (1997).

[14] A. V. Kyrylyuk, M. C. Hermant, T. Schilling, B. Klumperman, C. E. Koning, and P. van der Schoot, Nat. Nanotechnol. 6, 364 (2011).

[15] B. Vigolo, C. Coulon, M. Maugey, C. Zakri, and P. Poulin, Science 309, 920 (2005).

[16] A. P. Chatterjee, J. Phys.: Condens. Matter 20, 255250 (2008).

[17] R. H. J. Otten and P. van der Schoot, Phys. Rev. Lett. 103, 225704 (2009); J. Chem. Phys. 134, 094902 (2011).

[18] I. Balberg, N. Binenbaum, and N. Wagner, Phys. Rev. Lett. 52, 1465 (1984); I. Balberg and N. Binenbaum, Phys. Rev. A 35, 5174 (1987).

[19] Z. Neda, R. Florian, and Y. Brechet, Phys. Rev. E 59, 3717 (1999).
[20] M. Foygel, R. D. Morris, D. Anez, S. French, and V. L. Sobolev, Phys. Rev. B 71, 104201 (2005).

[21] L. Berhan and A. M. Sastry, Phys. Rev. E 75, 041120 (2007).

[22] T. Schilling, S. Jungblut, and M. A. Miller, Phys. Rev. Lett. 98, 108303 (2007).

[23] G. Ambrosetti, C. Grimaldi, I. Balberg, T. Maeder, A. Danani, and P. Ryser, Phys. Rev. B 81, 155434 (2010).

[24] M. Allen, G. Evans, D. Frenkel, and B. Mulder, Adv. Chem. Phys. 86, 1 (1993).

[25] P. G. de Gennes and J. Prost, The Physics of Liquid Crystals (Oxford University Press, Oxford, 1995).

[26] B. Nigro and C. Grimaldi (unpublished).

[27] B. Nigro, G. Ambrosetti, C. Grimaldi, T. Maeder, and P. Ryser, Phys. Rev. B 83, 064203 (2011).

[28] B. Nigro, C. Grimaldi, M. A. Miller, P. Ryser, and T. Schilling, J. Chem. Phys. 136, 164903 (2012).

[29] R. Fogelholm, J. Phys. C 13, L571 (1980).

[30] G. G. Batrouni and A. Hansen, J. Stat. Phys. 52, 747 (1988).

[31] G. H. Golub and C. F. Van Loan, Matrix Computations (Johns Hopkins University Press, Baltimore, 1996).

[32] N. Johner, Ph.D. thesis No. 4351, Ecole Polytechnique Fédérale de Lausanne (EPFL), 2009.

[33] V. Ambegaokar, B. I. Halperin, and J. S. Langer, Phys. Rev. B 4, 2612 (1971); M. Pollak, J. Non-Cryst. Solids 11, 1 (1972); B. I. Shklovskii and A. L. Efros, Sov. Phys. JETP 33, 468 (1971); 34, 435 (1972).

[34] B. Nigro, C. Grimaldi, P. Ryser, A. P. Chatterjee, and P. van der Schoot, Phys. Rev. Lett. 110, 015701 (2013).

[35] B. Nigro, C. Grimaldi, P. Ryser, F. Varrato, G. Foffi, and P. J. Lu, Phys. Rev. E 87, 062312 (2013).

[36] C. Grimaldi, Europhys. Lett. 96, 36004 (2011).

[37] F. Y. Wu, J. Phys. A 37, 6653 (2004).

[38] T. Boublík, Mol. Phys. 32, 1737 (1976).

[39] C. Vega and D. Frenkel, Mol. Phys. 67, 633 (1989). 